\title{
Matching-adjusted indirect comparisons of efficacy of BAY 8I-8973 vs two recombinant factor VIII for the prophylactic treatment of severe hemophilia $A$
}

\author{
This article was published in the following Dove Press journal: \\ Journal of Blood Medicine \\ 4 July 2016 \\ Number of times this article has been viewed
}

\author{
Jennifer Pocoski' \\ Nanxin $\mathrm{Li}^{2}$ \\ Rajeev Ayyagari ${ }^{2}$ \\ Nikki Church' \\ Monika Maas Enriquez' \\ Quer Xiang ${ }^{2}$ \\ Sneha Kelkar ${ }^{3}$ \\ Ella $X \mathrm{Du}^{2}$ \\ Eric $\mathrm{Q} \mathrm{Wu}^{2}$ \\ Jipan $\mathrm{Xie}^{3}$
}

'Bayer HealthCare Pharmaceuticals, Whippany, NJ, ${ }^{2}$ Analysis Group, Inc., Boston, MA, ${ }^{3}$ Analysis Group, Inc., New York, NY, USA
Correspondence: Nanxin Li Analysis Group, Inc., I I Huntington Avenue, 14th Floor, Boston, MA 02199, USA

Tel +l 6174258478

Email nick.li@analysisgroup.com
Background: No head-to-head trials comparing recombinant factor VIII (rFVIII) products currently exist. This was a matching-adjusted indirect comparison (MAIC) study of efficacy of BAY 81-8973 with antihemophilic factor (recombinant) plasma/albumin-free method (rAHFPFM) and turoctocog alfa for the prophylaxis of severe hemophilia A.

Methods: A systematic literature review was conducted to identify trials of rAHF-PFM and turoctocog alfa. Comparisons were conducted using BAY 81-8973 individual patient data (IPD) from LEOPOLD trials and published data from rAHF-PFM and turoctocog alfa trials. Differences in outcome reporting were reconciled using transformation of BAY 81-8973 IPD. Patients in pooled LEOPOLD trials were weighted to match baseline characteristics for $\mathrm{AAHF}$ PFM or turoctocog alfa trials using MAICs. After matching, annualized bleed rates (ABRs) were compared using weighted $t$-tests.

Results: Two rAHF-PFM trials and one turoctocog alfa trial were identified. In these trials, rFVIIIs were dosed thrice weekly or every other day; in LEOPOLD trials, BAY 81-8973 was dosed twice- or thrice weekly. Three MAICs were conducted because the two rAHF-PFM trials calculated ABRs differently, matching for age, race, and weight (turoctocog alfa only). BAY 81-8973 had similar ABR of all bleeds vs rAHF-PFM (two trials: 4.8 vs 6.3, 1.9 vs 1.8 [square root transform]) and lower ABR of spontaneous bleeds and trauma bleeds (2.6 vs 4.1, 2.1 vs 4.7; both $P<0.05)$. BAY 81-8973 showed lower ABR of all bleeds and spontaneous bleeds vs turoctocog alfa (4.3 vs 6.5, 2.8 vs 4.3; both $P<0.05)$ and similar ABR of trauma bleeds (1.5 vs 1.6). In subgroup analysis, twice-weekly BAY 81-8973 had similar ABRs of all bleeds, spontaneous bleeds, and trauma bleeds compared to rAHF-PFM and turoctocog alfa.

Conclusion: This indirect comparison found that prophylaxis with BAY 81-8973, even including the lower frequency of two times a week and lower factor VIII consumption, has efficacy comparable to rAHF-PFM and turoctocog alfa, which were dosed thrice weekly or every other day. The use of IPD enabled adjustments for differences in calculation of ABRs and population characteristics between trials.

Keywords: hemophilia A, BAY 81-8973, rAHF-PFM, turoctocog alfa, MAIC, annualized bleed rate

\section{Background}

Hemophilia A is the result of missing, or reduced, clotting factor VIII (FVIII) in the circulating blood, characterized by excessive bleeding into joints and soft tissues from sites experiencing trauma or spontaneous bleeds. ${ }^{1}$ Approximately $60 \%$ of all affected 
individuals have severe hemophilia A, defined as $<1 \%$ plasma FVIII activity. ${ }^{2}$ To reduce the risk of bleeding and chronic arthropathy, World Federation of Hemophilia guidelines recommend prophylaxis with FVIII products over on-demand treatment for patients with severe hemophilia A to maintain FVIII levels $>1 \%$. $^{3}$

Currently, prophylaxis with third-generation unmodified recombinant factor VIII (rFVIII) is the mainstay of treatment. BAY 81-8973 (Kovaltry ${ }^{\circledR}$; Bayer HealthCare Pharmaceuticals, Whippany, NJ, USA) is a full-length human rFVIII that is currently under US Food and Drug Administration and European Medicines Agency review. Multiple clinical trials have demonstrated the efficacy and safety of BAY 81-8973 for the prophylaxis of severe hemophilia A. ${ }^{4}$ Antihemophilic factor (recombinant) plasma/albumin-free method (rAHFPFM; Advate ${ }^{\circledR}$, Baxter Healthcare Corporation, Mississauga, ON, Canada), is an unmodified rFVIII approved in 2003; turoctocog alfa $\left(\right.$ NovoEight $^{\circledR}$, Novo Nordisk Inc, Plainsboro, NJ, USA), approved in 2013, was the first new rFVIII approved in this decade. ${ }^{5,6}$ Both of them are commonly used as prophylactic treatments for patients with severe hemophilia A. ${ }^{5,6}$

With multiple unmodified rFVIII products available, the comparative efficacy of different products plays an important role in clinical and health economic decision making. However, no head-to-head trials comparing rFVIII products among patients with hemophilia A currently exist. The present study aimed to address such an evidence gap by indirectly comparing the efficacy of BAY 81-8973 with rAHF-PFM and turoctocog alfa. First, a systematic literature review (SLR) was performed to identify published clinical trials with information on the efficacy of rAHF-PFM and turoctocog alfa. Next, an indirect comparison of the efficacy of these rFVIII products was performed using published data from rAHFPFM and turoctocog alfa trials identified from the SLR and individual patient data (IPD) from BAY 81-8973 LEOPOLD I and II trials. Using transformation of BAY 81-8973 trial IPD, the current study investigators were able to overcome the challenges associated with naïve comparisons of trial results in this field and reconcile the differences in calculating and reporting of efficacy outcomes. A matching-adjusted indirect comparison (MAIC) approach, which enables adjusted indirect comparisons without common comparators (such as the case with these rFVIII trials) $)^{7-9}$ and has been used to support health technology assessment submissions based on single-arm trials, ${ }^{10,11}$ was used to compare the efficacy of BAY 81-8973 with rAHF-PFM and turoctocog alfa.

\section{Methods}

\section{Systematic literature review}

An SLR was conducted to identify clinical trials of rAHF-PFM and turoctocog alfa for the treatment of severe hemophilia A. The SLR was designed, performed, reported according to the Preferred Reporting Items for Systematic Reviews and MetaAnalysis guidelines ${ }^{12}$ and Haute Autorité de Santé guidelines for indirect comparisons, ${ }^{13}$ and conducted on March 31, 2015, using three electronic databases: EMBASE, MEDLINE and MEDLINE In-Process, and Cochrane Central Register of Controlled Trials. Two levels of screening were performed: Level I on abstract and title and Level II on full text. Studies were included if they were randomized or single-arm clinical trials of rAHF-PFM or turoctocog alfa among adult and adolescent patients with severe hemophilia $\mathrm{A}$, and reported efficacy outcomes for these treatments. Only Englishlanguage articles were included. Papers in other languages, publication types like review articles, study types such as retrospective studies or case reports, study populations other than hemophilia A, or study interventions other than rAHFPFM and turoctocog alfa were excluded. Detailed screening criteria are presented in Supplementary materials (Table S1). Two researchers reviewed all the publications independently in parallel. Any disagreements were resolved by discussions with or by independent arbitration from a third reviewer. For each comparison, the efficacy outcomes that could be compared between the trials were identified. For the included studies, information on study design and data on baseline characteristics and outcomes were extracted into a data collection spreadsheet with prepared fields.

\section{Data sources for indirect comparisons}

Where possible, IPD was obtained from the study investigators; where IPD was not available, aggregate results from the identified publications were extracted for the comparison.

For BAY 81-8973, IPD from LEOPOLD I (NCT01029340) ${ }^{14}$ and LEOPOLD II (NCT01233258) ${ }^{15}$ trials was available. LEOPOLD I was a two-part study: Part A was a Phase I study that evaluated the concentration time and half-life of BAY 81-8973; Part B, a Phase II/III trial, assessed the bleeding outcomes. LEOPOLD II was a Phase II/III trial that reported bleeding outcomes. Prophylaxis arms in Part B of LEOPOLD I and in LEOPOLD II were pooled for the analysis. For rAHF-PFM and turoctocog alfa, IPD was not available for this comparison. Published aggregate results from included studies based on the SLR were used.

The efficacy outcomes considered included annualized bleed rates (ABRs) for all bleeds, spontaneous bleeds, and 
trauma bleeds when available, as well as proportion of patients with zero bleeds. They were measured at 1 year and also at 6 months intervals in the LEOPOLD I and II trials.

\section{Indirect comparisons}

Differences in populations, design, or outcome reporting across trials, such as the method of computation of bleeding rates or trial lengths, were reviewed in detail and reconciled to the extent possible by making adjustments using the IPD from LEOPOLD I and II trials accordingly. When more than one prophylaxis arm was present in a published trial, they were pooled for the analysis. The efficacy outcomes reported at comparable time points in both BAY 81-8973 trials, and comparator trials were identified and selected for comparison.

Comparisons were performed separately for BAY 81-8973 vs rAHF-PFM and BAY 81-8973 vs turoctocog alfa. MAICs were used to match populations based on published aggregates for baseline characteristics. The pooled prophylaxis arms from the BAY 81-8973 trials were reweighted such that the average baseline characteristics matched the corresponding published characteristics in the comparator trials, making the population of BAY 81-8973 trials more similar to the comparator trials. After matching baseline characteristics, efficacy outcomes were compared between BAY 81-8973 and rAHF-PFM or turoctocog alfa using weighted $t$-tests.

BAY 81-8973 was dosed either two or three times a week in LEOPOLD I and II trials. A subgroup analysis was conducted comparing the low-dose two times a week regimen of BAY 81-8973 with rAHF-PFM or turoctocog alfa, both of which were dosed three times a week or every other day, using similar methods as those already described for the overall patient population.

Statistical significance was assessed at 0.05 levels. All statistical analyses were conducted using SAS v9.3.

\section{Results}

\section{SLR and comparison of study designs}

The SLR identified 82 unique publications for screening. After two levels of screening (Figure 1), three publications were

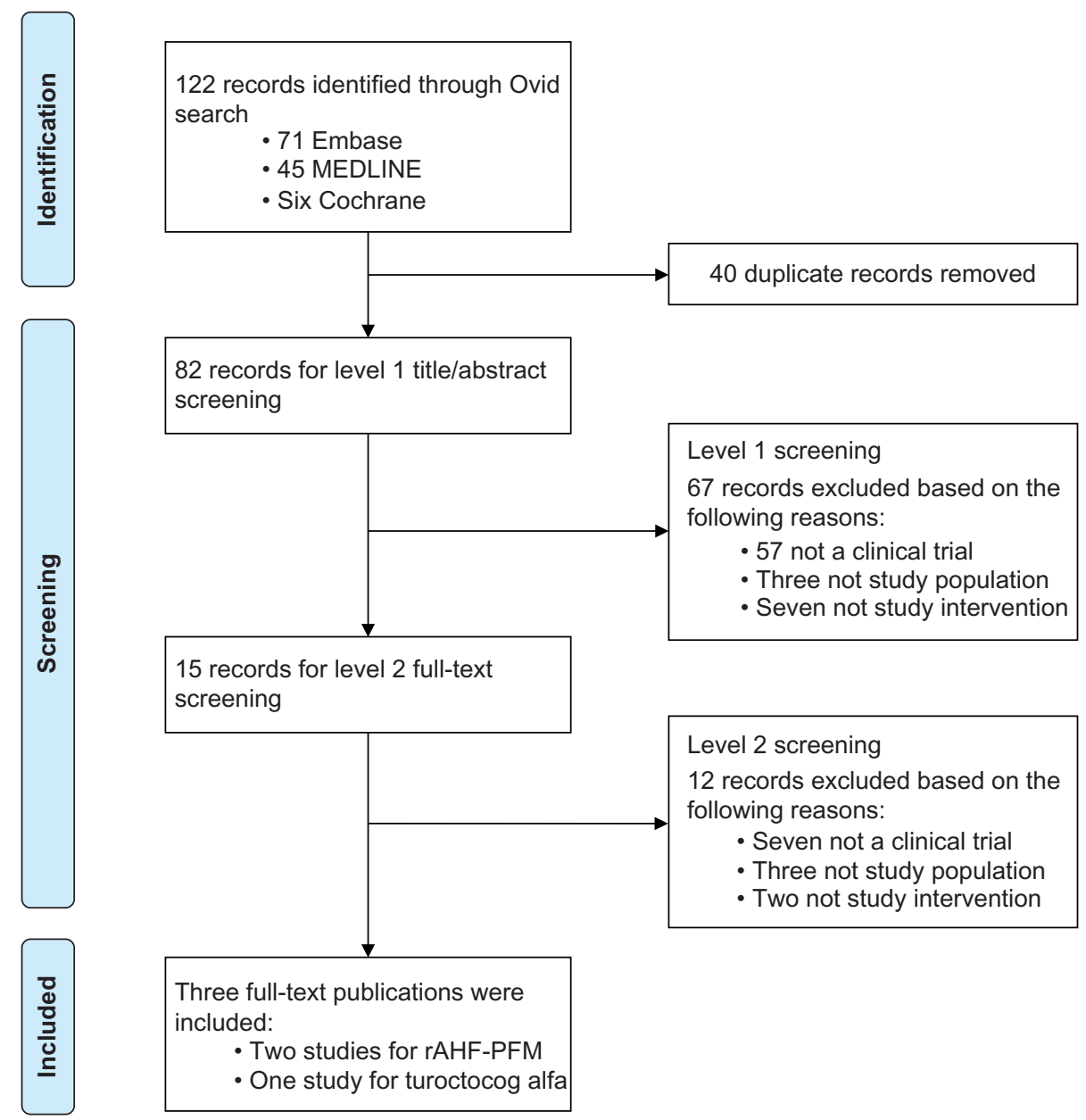

Figure I PRISMA diagram for SLR.

Abbreviations: ABRs, annualized bleed rates; PRISMA, Preferred Reporting Items for Systematic Reviews and Meta-Analysis; rAHF-PFM, antihemophilic factor (recombinant) plasma/albumin-free method; SLR, systematic literature review. 
included for indirect comparisons: two trials for rAHF-PFM (Tarantino et $\mathrm{al}^{16}$ and Valentino et $\mathrm{al}^{17}$ ) and one trial for turoctocog alfa (Lentz et $\mathrm{al}^{18}$ ). Prophylaxis arms for the two rAHFPFM trials could not be pooled because of inherent differences in ABR calculations. Therefore, three MAICs were conducted subsequently: BAY 81-8973 vs rAHF-PFM (Valentino et al), ${ }^{17}$ BAY 81-8973 vs rAHF-PFM (Tarantino et al), ${ }^{16}$ and BAY 81-8973 vs turoctocog alfa.

Only the baseline characteristics that were reported across both the LEOPOLD I or II and rAHF-PFM (Valentino et al), ${ }^{17}$ and rAHF-PFM (Tarantino et al), ${ }^{16}$ or turoctocog alfa trials could potentially be matched during MAIC. Reported baseline characteristics included age, race composition, or weight for the rAHF-PFM and turoctocog alfa trials. Baseline characteristics, such as comorbidities, Gilbert score, target joint for bleeds, and number of bleeds in the past year, could not be matched because they were available only in the BAY 81-8973 trials. In addition, both rAHF-PFM trials included a mix of severe and moderately severe hemophilia A, but only reported pooled results among all patients. In contrast, BAY 81-8973 trials only included patients with severe hemophilia $A$, and thus could not adjust for such inclusion of moderately severe patients in rAHFPFM trials. Baseline characteristics before matching differed vastly across trials. In the rAHF-PFM (Valentino et al) ${ }^{17}$ trial, $86 \%$ of patients were aged $>16$ years, $88 \%$ were White, and $2 \%$ were Asian. In the rAHF-PFM (Tarantino et al) ${ }^{16}$ trial, $50 \%$ of patients were aged $>18$ years, $93 \%$ were White, and only $1 \%$ were Asian. In the turoctocog alfa trial, the mean age was 28 years, the mean weight was $73 \mathrm{~kg}, 81 \%$ of patients were White, and $13 \%$ were Asian. In the pooled BAY 81-8973 trials, $91 \%$ of patients were aged $>16$ years, $83 \%$ were aged $>18$ years, the mean age was 30 years, the mean weight was $71 \mathrm{~kg}$, only $70 \%$ were White, and as many as $19 \%$ were Asian.

To ensure fair comparisons between BAY 81-8973, rAHF-PFM, and turoctocog alfa, data transformations of BAY 81-8973 trial data were needed. For comparison of BAY 81-8973 vs rAHF-PFM (Valentino et al), ${ }^{17}$ the ABRs reported by Valentino et $\mathrm{al}^{17}$ were transformed using a square root function: $\mathrm{ABR}^{\prime}=\sqrt{\mathrm{ABR}+0.5}$. Hence, the same transformation was applied to the IPD of BAY 81-8973. Tarantino et $\mathrm{al}^{16}$ did not report the estimates for standard deviation (SD) or standard error associated with the mean ABRs. To determine statistical significance for the comparisons, SDs obtained in Tarantino et al's study ${ }^{16}$ were imputed per recommendations from Cochrane handbook ${ }^{19}$ by using the same SDs as for the ABRs from the BAY 81-8973 trials. The impact of this assumption was tested in sensitivity analyses using 0.5 times or 1.5 times the SDs from the BAY 81-8973 trials.

The duration of prophylaxis was 1 year in BAY 81-8973 (both LEOPOLD I and II) and rAHF-PFM (Valentino et al) ${ }^{17}$ trials and 6 months in rAHF-PFM (Tarantino et al) ${ }^{16}$ and turoctocog alfa trials. Therefore, for BAY 81-8973 vs rAHF-PFM (Valentino et al), ${ }^{17} 1$-year outcomes were compared; for BAY 81-8973 vs rAHF-PFM (Tarantino et al) ${ }^{16}$ and BAY 81-8973 vs turoctocog alfa, both 1-year and the first 6-month outcomes were compared.

\section{Baseline characteristics}

A total of 62 and 59 intent-to-treat patients from the prophylaxis arms of the LEOPOLD I and II trials, respectively, were included. The reported trial population of $\mathrm{rAHF}-\mathrm{PFM}$ (Valentino et al), ${ }^{17}$ rAHF-PFM (Tarantino et al), ${ }^{16}$ and turoctocog alfa included 66,107 , and 150 patients receiving prophylactic treatment, respectively.

Before matching, there were significant differences in comparable baseline characteristics for all three comparisons. For BAY 81-8973 vs rAHF-PFM (Valentino et al), ${ }^{17}$ the BAY 81-8973 trials had similar proportions of patients with age $\geq 16$ (91\% vs $86 \%, P=0.34)$, higher proportion of patients of Asian race $(19 \%$ vs $2 \%, P<0.05)$, and lower proportion of White patients $(70 \%$ vs $88 \%, P<0.05)$. For BAY 81-8973 vs rAHF-PFM (Tarantino et al), ${ }^{16}$ proportions of patients with age $>18$ years and Asian race were higher ( $80 \%$ vs $53 \%$ and $19 \%$ vs $1 \%$, both $P<0.01$ ), and proportion of White patients was lower $(70 \%$ vs $93 \%, P<0.01)$ in BAY 81-8973 trials. For BAY 81-8973 vs turoctocog alfa, patients in BAY 81-8973 trials were similar in age, weight, and proportion of Asian patients (mean age: 30 vs 28 years, $P=0.12$; mean weight: $71 \mathrm{~kg}$ vs $73 \mathrm{~kg}, P=0.32$; Asian race: $19 \%$ vs $13 \%, P=0.20)$, with a lower proportion of White race $(70 \%$ vs $81 \%, P<0.05)$.

After matching, all average baseline characteristics were balanced between BAY 81-8973 and each of the comparator trials (all $P=1.00)$.

\section{Indirect comparisons BAY 8I-8973 vs rAHF-PFM}

I-year results (only)

Approximately 6\% fewer patients in BAY 81-8973 trials experienced zero bleeds before and after matching as compared to rAHF-PFM (Valentino et al) ${ }^{17}$ trial (both $27 \%$ vs $33 \%, P>0.05)$. The square root transformed ABR of all bleeds for BAY 81-8973 and rAHF-PFM was similar before and after matching (both 1.9 vs $1.8, P>0.05$; Figure 2 ). 


\section{BAY $8 \mathrm{I}-8973$ vs rAHF-PFM}

\section{I-year results}

Based on comparison of 1-year outcomes, a similar proportion of patients in BAY 81-8973 trials experienced zero bleeds as compared with rAHF-PFM (Tarantino et al) ${ }^{16}$ trial $(27 \%$ vs $30 \%, P=0.66$ ) before matching. After matching, the differences remained similar ( $26 \%$ vs $30 \%, P=0.54$ ). Compared to rAHF-PFM (Tarantino et al) ${ }^{16}$ trial, before matching, the ABRs for all bleeds ( 4.4 vs $6.3, P<0.05$ ), spontaneous bleeds ( $3.0 \mathrm{vs}$ $4.1, P=0.09$ ), and trauma bleeds ( 1.3 vs $4.7, P<0.05$ ) were lower in BAY 81-8973 trials. After matching, the ABR difference for all bleeds did not reach statistical significance $(-1.5 ; P=0.18)$, but ABRs for spontaneous and trauma bleeds in BAY 81-8973 trials remained lower with differences of $-1.5(P<0.05)$ and $-2.6(P<0.05)$, respectively (Figure 3 ).
The sensitivity analyses based on varying the imputed SD of rAHF-PFM (Tarantino et al) ${ }^{16}$ trial by 0.5 and 1.5 times of SD from the BAY 81-8973 trials showed similar results as the base case ( 0.5 times: all bleeds, $P=0.14$; spontaneous bleeds, $P<0.05$; trauma bleeds, $P<0.05 ; 1.5$ times: all bleeds, $P=0.25$; spontaneous bleeds, $P=0.09$; trauma bleeds, $P<0.05$; the mean differences were the same as the base case because varying SDs would only affect $P$-value estimates).

\section{First 6-month results}

During the first 6 months, $36 \%$ and $38 \%$ of the patients in BAY 81-8973 trials experienced zero bleeds, compared to $30 \%$ in the rAHF-PFM (Tarantino et al) ${ }^{16}$ trial before and after matching (both $P>0.05$ ). Compared with rAHF-PFM (Tarantino et al) ${ }^{16}$ trial, before matching, the ABRs for all

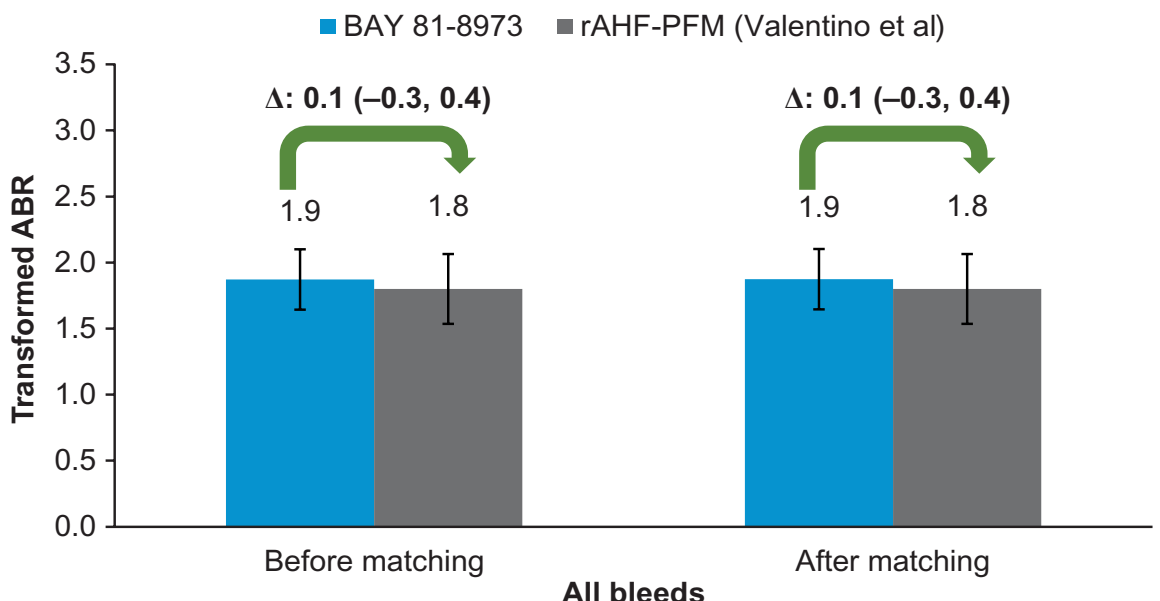

Figure 2 ABRs for BAY 8I-8973 (I-year) vs rAHF-PFM.

Note: Data from Valentino et al. ${ }^{17}$

Abbreviations: ABRs, annualized bleed rates; rAHF-PFM, antihemophilic factor (recombinant) plasma/albumin-free method.

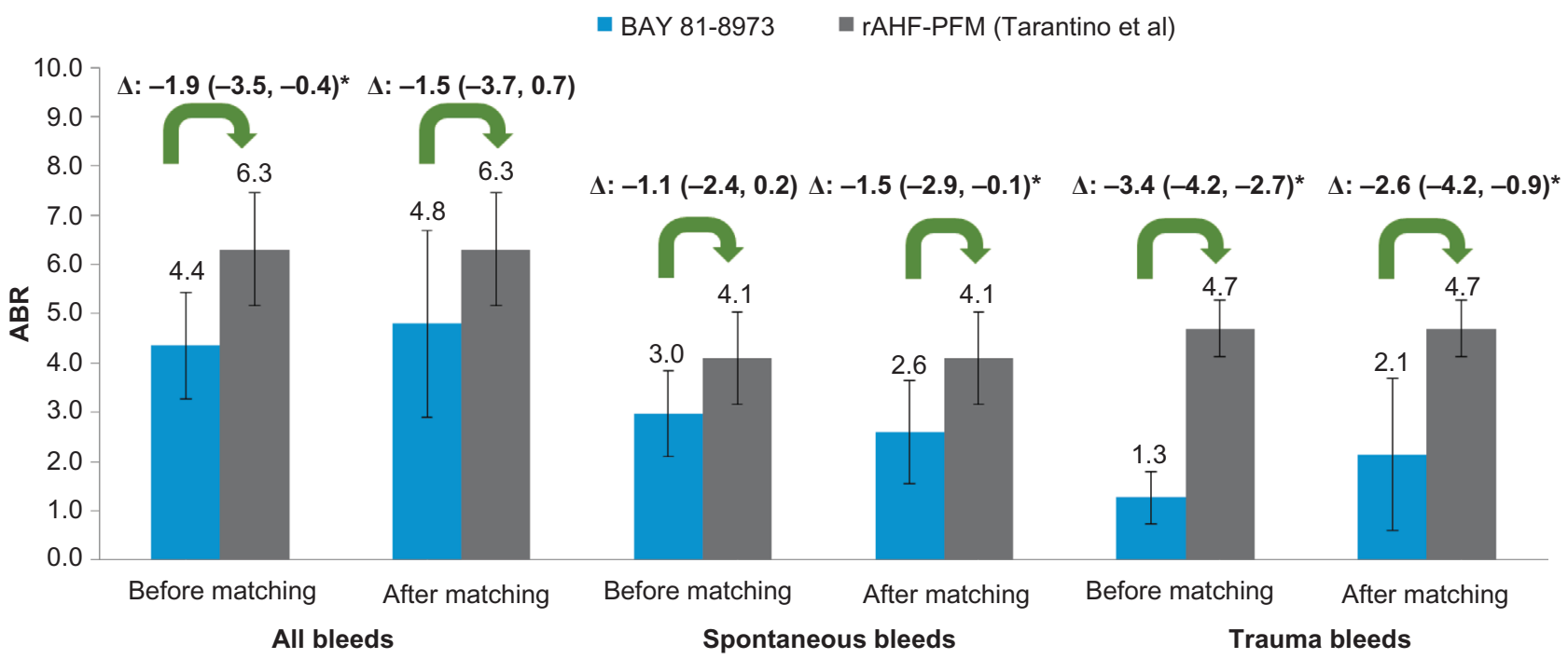

Figure 3 ABRs for BAY $81-8973$ (I-year) vs rAHF-PFM.

Notes: $* P<0.05$. Data from Tarantino et al. ${ }^{16}$

Abbreviations: ABRs, annualized bleed rates; rAHF-PFM, antihemophilic factor (recombinant) plasma/albumin-free method. 
bleeds ( 5.2 vs $6.3, P=0.27)$ and spontaneous bleeds (3.6 vs 4.1, $P=0.57$ ) were numerically lower, and the ABR for trauma bleeds ( 1.4 vs $4.7, P<0.01)$ was significantly lower in BAY 81-8973 trials. After matching, the ABRs in BAY 81-8973 trials compared to rAHF-PFM (Tarantino et al) ${ }^{16}$ trial were 5.5 vs $3.3(P=0.51)$ for all bleeds, $3.0 \%$ vs $4.1 \%(P=0.23)$ for spontaneous bleeds and were significantly lower with a difference of $-2.4(P<0.05)$ for all trauma bleeds (Table 1$)$. Consistent with 1-year results, the sensitivity analyses based on varying the imputed SD of rAHF-PFM (Tarantino et al) ${ }^{16}$ trial showed similar results as the base case.

\section{BAY 8I-8973 vs turoctocog alfa}

\section{$\mathrm{I}$-year results}

Based on comparison of 1-year outcomes, 27\% vs 30\% of patients experienced zero bleeds with BAY 81-8973 as compared to turoctocog alfa before matching $(P=0.62)$, whereas after matching, the corresponding proportions were $26 \%$ vs $30 \%(P=0.47)$. Compared to turoctocog alfa trial, before matching, the ABRs for all bleeds (4.4 vs 6.5,
$P<0.01)$, spontaneous bleeds ( 3.0 vs $4.3, P<0.05)$ were significantly lower, while the ABR for trauma bleeds was 1.3 vs $1.6(P=0.22)$. After matching, the ABRs for all bleeds and spontaneous bleeds in BAY 81-8973 trials remained significantly lower with differences of $-2.2(P<0.05)$ and $-1.5(P<0.05)$. The ABR for trauma bleeds was still similar after matching $(-0.1 ; P=0.69)$ (Figure 4$)$.

\section{First 6-month results}

Based on comparison of first 6-month outcomes, a numerically higher proportion of BAY 81-8973-treated patients experienced zero bleeds as compared to turoctocog alfa before and after matching (before: $36 \%$ vs $30 \%, P=0.27$; after: $37 \%$ vs $30 \%, P=0.26)$. Before matching, the ABRs for all bleeds ( 5.2 vs $6.5, P=0.07$ ), spontaneous bleeds ( 3.6 vs $4.3, P=0.25$ ), and trauma bleeds ( 1.4 vs $1.6, P=0.61)$ were comparable between BAY 81-8973 and turoctocog alfa. After matching, the differences of ABRs remained similar between BAY 81-8973 and turoctocog alfa (all bleeds: $-1.4, P<0.05$; spontaneous bleeds: $-1.0, P=0.08$; trauma bleeds: $0.0, P=0.98$; Table 2 ).

Table I Outcomes for BAY 8I-8973 (first 6-month) vs rAHF-PFM

\begin{tabular}{|c|c|c|c|c|c|c|}
\hline \multirow[t]{3}{*}{ Outcomes } & \multicolumn{3}{|c|}{ Before matching } & \multicolumn{3}{|c|}{ After matching } \\
\hline & BAY 8I-8973 & rAHF-PFM & Difference & BAY 8I-8973 & rAHF-PFM & Difference \\
\hline & $(N=121)$ & $(N=107)$ & & $(N=|2|)$ & $(N=107)$ & \\
\hline Patients with zero bleeds, \% & 36 & 30 & 6 & 38 & 30 & 8 \\
\hline \multicolumn{7}{|l|}{$A B R$, mean $(S D)$} \\
\hline All bleeds & $5.2(7.3)$ & $6.3(7.3)$ & -1.1 & $5.4(7.7)$ & $6.3(7.3)$ & -0.9 \\
\hline Spontaneous bleeds & $3.6(6.4)$ & 4.I (6.4) & -0.5 & $3.0(5.6)$ & $4.1(6.4)$ & -1.1 \\
\hline Trauma bleeds & $1.4(3.7)$ & $4.7(3.7)$ & $-3.3^{*}$ & $2.3(5.4)$ & $4.7(3.7)$ & $-2.4^{*}$ \\
\hline
\end{tabular}

Notes: $* P<0.05$. Data from Tarantino et al. ${ }^{16}$

Abbreviations: $A B R$, annualized bleed rate; SD, standard deviation.

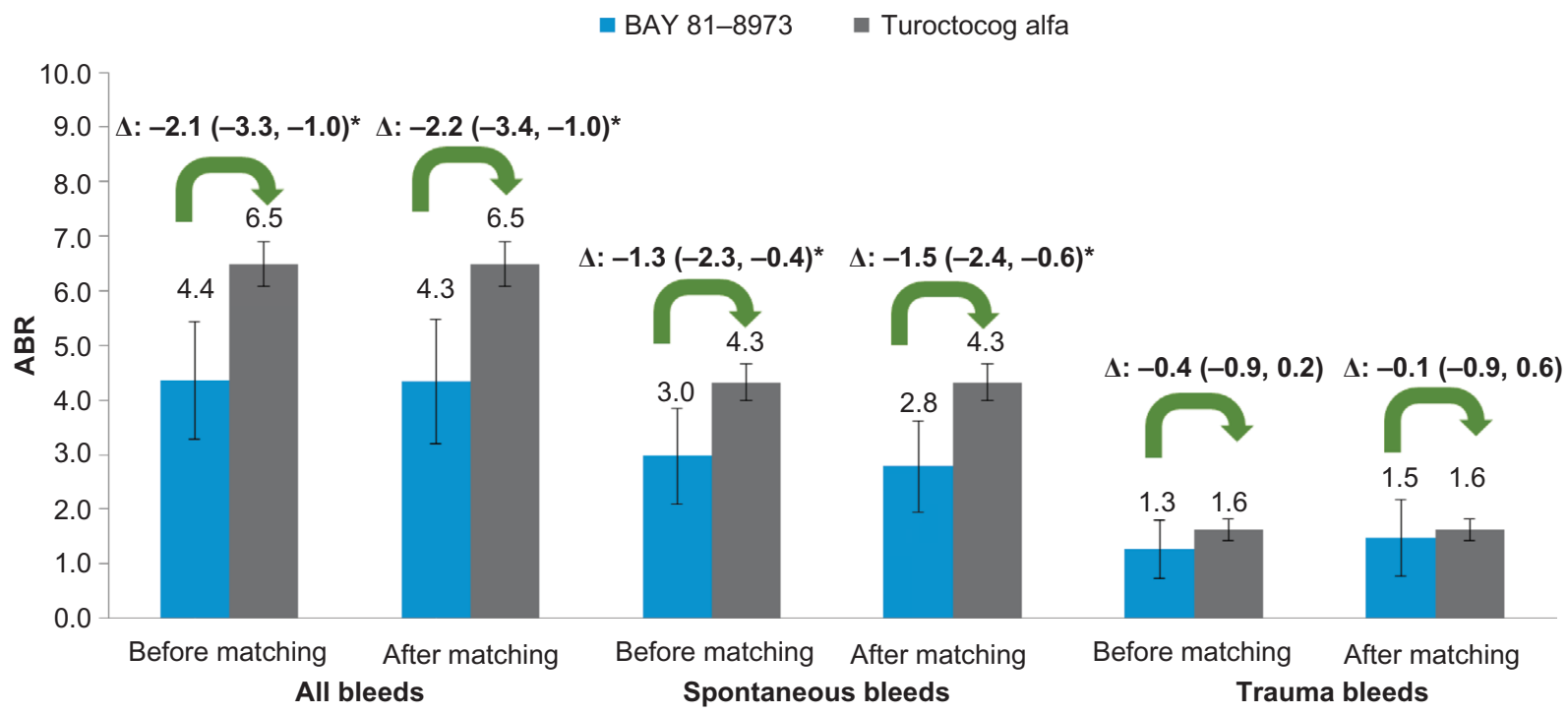

Figure 4 ABRs for BAY $8 \mathrm{I}-8973$ ( 1 -year) vs turoctocog alfa.

Notes: $* P<0.05$. Data from Lentz et al. ${ }^{18}$

Abbreviation: ABRs, annualized bleed rates. 
Table 2 Outcomes for BAY 8I-8973 (first 6-month) vs turoctocog alfa

\begin{tabular}{|c|c|c|c|c|c|c|}
\hline \multirow[t]{3}{*}{ Outcome } & \multicolumn{3}{|c|}{ Before matching } & \multicolumn{3}{|c|}{ After matching } \\
\hline & \multirow{2}{*}{$\begin{array}{l}\text { BAY 8I-8973 } \\
(\mathrm{N}=12 \mathrm{I})\end{array}$} & \multirow{2}{*}{$\begin{array}{l}\text { Turoctocog alfa } \\
(N=150)\end{array}$} & \multirow[t]{2}{*}{ Difference } & \multirow{2}{*}{$\begin{array}{l}\text { BAY 8I-8973 } \\
(\mathrm{N}=121)\end{array}$} & \multirow{2}{*}{$\begin{array}{l}\text { Turoctocog alfa } \\
(N=150)\end{array}$} & \multirow[t]{2}{*}{ Difference } \\
\hline & & & & & & \\
\hline Patients with zero bleeds, \% & 36 & 30 & 6 & 37 & 30 & 7 \\
\hline \multicolumn{7}{|l|}{$A B R$, mean $(S D)$} \\
\hline All bleeds & $5.2(7.3)$ & $6.5(2.5)$ & -1.3 & $5.1(7.1)$ & $6.5(2.5)$ & $-1.4 *$ \\
\hline Spontaneous bleeds & $3.6(6.4)$ & $4.3(2.1)$ & -0.7 & $3.3(6.0)$ & $4.3(2.1)$ & -1.0 \\
\hline Trauma bleeds & $\mathrm{I} .4(3.7)$ & $1.6(1.3)$ & -0.2 & I.6 (4.0) & $1.6(1.3)$ & 0.0 \\
\hline
\end{tabular}

Notes: $* P<0.05$. Data from Lentz et al. ${ }^{18}$

Abbreviations: $A B R$, annualized bleed rate; $S D$, standard deviation.

\section{Subgroup analyses}

Subgroup analyses were conducted comparing the lowdose twice-weekly regimen of BAY 81-8973 with rAHFPFM or turoctocog alfa, both of which were dosed thrice weekly or every other day. A total of 46 patients from LEOPOLD I $(\mathrm{N}=18)$ and II trials $(\mathrm{N}=28)$ were included. The detailed subgroup analyses results are summarized in Supplementary materials (Figures S1-S3 and Tables S2 and S3). Briefly, compared to rAHF-PFM, twice-weekly BAY 81-8973 had similar ABRs of all bleeds (6.1 vs 6.3 for rAHF-PFM [Tarantino et al] ${ }^{16}$ trial and 2.0 vs 1.8 for rAHF-PFM [Valentino et al] ${ }^{17}$ trial), spontaneous bleeds (3.4 vs 4.1 for rAHF-PFM [Tarantino et al] ${ }^{16}$ trial), and trauma bleeds (2.6 vs 4.7 for rAHF-PFM [Tarantino et al] ${ }^{16}$ trial). Compared to turoctocog alfa, twice-weekly BAY 81-8973 had similar ABRs of all bleeds (5.2 vs 6.5), spontaneous bleeds (3.4 vs 4.3$)$, and trauma bleeds (1.7 vs 1.6$)(P>0.05$ for all comparisons). While LEOPOLD I allowed twiceweekly BAY 81-8973 at physician's discretion, LEOPOLD II study randomly assigned patients to receive twice-weekly BAY 81-8973. To address potential bias because of dosing at physician's discretion, two subgroup analyses were conducted based on LEOPOLD II alone: the first compared both BAY 81-8973 prophylaxis arms with rAHF-PFM or turoctocog alfa, and the second compared the low-dose twice-weekly prophylaxis arm with these treatments. The results, detailed in Supplementary materials (Tables S4-S13), were consistent with those using pooled LEOPOLD I and LEOPOLD II studies.

\section{Discussion}

This MAIC analysis found that prophylactic treatment with BAY 81-8973, including the thrice-weekly regimen as well as the lower-dose twice-weekly regimen, demonstrated efficacy comparable to rAHF-PFM and turoctocog alfa, which were dosed thrice weekly or every other day, with a range of differences from -2.6 to 0.1 in ABRs and from $-6 \%$ to $8 \%$ in proportion of patients with zero bleeds. An assessment of the trials identified that the studies were broadly similar in design, although there were differences in the durations and methods of assessment of the ABRs. Some differences in the population characteristics at baseline were also identified. The use of IPD from the LEOPOLD trials enabled the analysis to address differences in the durations and methods of calculation of ABRs between trials and also permitted adjustment for some cross-trial differences in baseline characteristics between trials (to the extent that they were reported in both BAY 81-8973 and comparator trials).

Clinical trials in hemophilia A are usually single-arm studies. There are no head-to-head randomized controlled trials (RCTs) directly comparing existing unmodified rFVIII products. In the absence of head-to-head RCTs, naïve comparisons of point estimates may provide a crude assessment of comparative effectiveness between different treatments. However, such naïve comparisons without comparator arms are discouraged by guidelines issued by leading health technology assessment agencies. ${ }^{13}$ Our study employed the MAIC method, which has been previously validated for comparative effectiveness research ${ }^{7-9}$ and used for analyses of single-arm trials. ${ }^{20}$ Using MAIC, the present study eliminated differences in commonly reported baseline characteristics between BAY 81-8973 and comparator trials, and thus led to indirect comparisons in more balanced patient populations. Furthermore, use of IPD from BAY 81-8973 trials allowed for the same square root transformation of $\mathrm{ABR}$ and analysis of outcomes from the first 6 months, which was used in the rAHF-PFM (Valentino et al) ${ }^{17}$ trial for the former and the rAHF-PFM (Tarantino et al $)^{16}$ and turoctocog alfa trials for the latter, thus enabling fair comparisons of the efficacy outcomes. While these adjustment methods are not substitutes for head-to-head RCTs, they are a significant improvement over a simple naïve comparison of the trial results.

In the current study, after matching on baseline characteristics, based on comparison of 1-year results, proportion 
of patients with zero bleeds were comparable among BAY 81-8973, rAHF-PFM, and turoctocog alfa. Compared to rAHFPFM, BAY 81-8973 showed similar ABR of all bleeds (mean differences for two trials: -1.5 and 0.1 , both $P>0.05$ ) and lower ABR of trauma bleeds and spontaneous bleeds (reported in one trial: -1.5 and -2.6 , both $P<0.05$ ). Compared to turoctocog alfa, BAY 81-8973 showed lower ABR for all bleeds and spontaneous bleeds $(-2.2$ and -1.5 , both $P<0.05)$ and similar ABR for trauma bleeds $(-0.1, P>0.05)$. The results were generally consistent across different sensitivity analyses (eg, first 6-month outcomes) and subgroups analyses (eg, BAY 81-8973 two times weekly). These results suggest that BAY 81-8973 overall shows comparable efficacy to two commonly used unmodified rFVIII products, suggesting that physicians and patients would have an additional potent choice in the treatment arsenal of rFVIII products upon the regulatory approval of BAY 81-8973.

The findings from subgroup analyses of BAY 81-8973 low-dose twice-weekly regimen are of particular interest and have important implications for health care decision makers. Both rAHF-PFM and turoctocog alfa are dosed thrice weekly or every other day. Therefore, twice weekly BAY 81-8973 is associated with lower average rFVIII utilization levels relative to $\mathrm{rAHF}-\mathrm{PFM}$ and turoctocog alfa ${ }^{4,16-18}$ but showed comparable efficacy as these two rFVIII treatments based on the current analyses. Less frequent dosing schedule and reduced consumption of rFVIII can lower the burden of patients and potentially reduce costs to payers.

This study is subject to a few limitations. Although the MAIC methodology makes scientifically sound comparison using the best available data, it is limited by the availability of published information for baseline characteristics from the comparator trials. Potential residual confounding could still exist because of unobserved cross-trial differences in patient characteristics that were not reported (and hence could not be adjusted for), including joint status, presence of target joints, and historic ABR before study entry. In addition, both rAHF-PFM trials included patients with a mix of severe and moderately severe hemophilia A but only reported pooled results among all patients, while BAY 81-8973 trials included only patients with severe hemophilia A. Hence, comparisons of BAY 81-8973 vs rAHF-PFM were conservative (ie, biased against BAY 81-8973), as patients with moderately severe disease would achieve better efficacy than patients with severe disease. Furthermore, all bleeds were defined more broadly in BAY 81-8973 and turoctocog alfa trials (spontaneous bleeds, trauma bleeds, and bleeds due to other reasons) than in rAHF-PFM trials (only spontaneous bleeds and trauma bleeds). Comparisons of BAY 81-8973 vs rAHF-PFM were again conservative because of this difference, but the impact from bleeds caused by other reasons would be small, as few bleeds belonged to this category in the BAY 81-8973 trials. Lastly, unlike most indirect comparisons, there was no common comparator arm between the trials. Although the observed characteristics were balanced between trials using weighting, a common comparator arm can provide additional protection against unobserved differences.

\section{Conclusion}

This indirect comparison study attempted to address the differences in calculation of ABRs and some trial population differences. The results showed that prophylaxis with BAY 81-8973, even including the lower frequency of two times a week and lower FVIII consumption, has efficacy comparable to rAHF-PFM and turoctocog alfa, which were dosed thrice weekly or every other day.

\section{Acknowledgments}

The current research was presented at 9th Annual Congress of the European Association for Haemophilia and Allied Disorders 2016, Malmö, Sweden conference on February $3-5,2016$, and the abstract has been published in Volume 22, Issue Supplement S2, pages 19-111, February 2016 of Haemophilia - The Official Journal of the World Federation of Hemophilia.

This study was funded by Bayer, which develops BAY 81-8973.

\section{Author contributions}

All authors contributed toward data analysis, drafting and critically revising the paper and agree to be accountable for all aspects of the work.

\section{Disclosure}

J Pocoski, N Church, and M Maas Enriquez are employees of Bayer and own stock or stock options. N Li, R Ayyagari, Q Xiang, S Kelkar, E Du, E Wu, and J Xie are employees of Analysis Group Inc., which has received consultancy fees from Bayer. The authors report no other conflicts of interest in this work.

\section{References}

1. Stonebraker JS, Bolton-Maggs PH, Soucie JM, Walker I, Brooker M. A study of variations in the reported haemophilia A prevalence around the world. Haemophilia. 2010;16:20-32.

2. Roth DA, Tawa NE Jr, O'Brien JM, Treco DA, Selden RF; Factor VIII Transkaryotic Therapy Study Group. Nonviral transfer of the gene encoding coagulation factor VIII in patients with severe hemophilia A. N Engl J Med. 2001;344:1735-1742.

3. Srivastava A, Brewer AK, Mauser-Bunschoten EP, et al. Guidelines for the management of hemophilia. Haemophilia. 2013;19:e1-e47. 
4. Kavakli K, Yang R, Rusen L, et al. Prophylaxis vs. on-demand treatment with BAY 81-8973, a full-length plasma protein-free recombinant factor VIII product: results from a randomized trial (LEOPOLD II). JThromb Haemost. 2015;13:360-369.

5. ADVATE $^{\circledR}$ (Antihemophilic Factor [Recombinant]) [package insert], 5, 2011. Ontario, Canada: Baxter Healthcare Corporation.

6. U.S. Food and Drug Administration. October 15, 2013 Approval Letter-Novoeight. http://www.fda.gov/BiologicsBloodVaccines/ BloodBloodProducts/ApprovedProducts/LicensedProductsBLAs/ FractionatedPlasmaProducts/ucm371095.htm. Accessed on May 5, 2016.

7. Signorovitch JE, Wu EQ, Yu AP, et al. Comparative effectiveness without head-to-head trials: a method for matching-adjusted indirect comparisons applied to psoriasis treatment with adalimumab or etanercept. Pharmacoeconomics. 2010;28:935-945.

8. Signorovitch JE, Sikirica V, Erder MH, et al. Matching-adjusted indirect comparisons: a new tool for timely comparative effectiveness research. Value Health. 2012;15:940-947.

9. Erder MH, Xie J, Signorovitch JE, et al. Cost effectiveness of guanfacine extended-release versus atomoxetine for the treatment of attention-deficit/hyperactivity disorder: application of a matching-adjusted indirect comparison. Appl Health Econ Health Policy. 2012;10:381-395.

10. Scottish Medicines Consortium. Bristol-Myers Squibb. Daclatasvir $30 \mathrm{mg}$ and $60 \mathrm{mg}$ film-coated tablets (Daklinza $\left.{ }^{\circledR}\right)$ SMC No. (1002/14). 10 October 2014. http:/www.scottishmedicines.org.uk/files/advice/ daclatasvir_Daklinza_FINAL_Oct_2014_Updated_30.10.14_for_website.pdf. Accessed on May 5, 2016.

11. Australian Government Department of Health - The pharmaceutical benefits scheme. EVEROLIMUS, tablets, $5 \mathrm{mg}$ and $10 \mathrm{mg}$, Afinitor ${ }^{\circledR}$, Novartis Pharmaceuticals Australia Pty Ltd. http:/www.pbs.gov.au/info/ industry/listing/elements/pbac-meetings/psd/2014-03/everolimus-rcc. Accessed on May 5, 2016
12. Moher D, Liberati A, Tetzlaff J, Altman DG, Group P. Preferred reporting items for systematic reviews and meta-analyses: the PRISMA statement. BMJ. 2009;339:b2535.

13. Haute Autorité de Santé. Indirect Comparisons Methods and Validity. Cedex, France: HAS; 2009.

14. Bayer. Trial to evaluate the efficacy and safety of a new full length Recombinant Human FVIII for hemophilia A (Leopold I). Available from: https://clinicaltrials.gov/ct2/show/NCT01029340. NLM Identifier: NCT01029340. Accessed April 13, 2016.

15. Bayer. A trial to compare prophylaxis therapy to on-demand therapy with a new full length recombinant FVIII in patients with severe hemophilia A (Leopold II). Available from: https://clinicaltrials.gov/ct2/show/results/ NCT01233258. NLM Identifier: NCT01233258.Accessed April 13, 2016.

16. Tarantino MD, Collins PW, Hay CR, et al. Clinical evaluation of an advanced category antihaemophilic factor prepared using a plasma/albumin-free method: pharmacokinetics, efficacy, and safety in previously treated patients with haemophilia A. Haemophilia. 2004;10:428-437.

17. Valentino LA, Mamonov V, Hellmann A, et al. A randomized comparison of two prophylaxis regimens and a paired comparison of on-demand and prophylaxis treatments in hemophilia A management. J Thromb Haemost. 2012;10:359-367.

18. Lentz SR, Misgav M, Ozelo M, et al. Results from a large multinational clinical trial (guardian1) using prophylactic treatment with turoctocog alfa in adolescent and adult patients with severe haemophilia A: safety and efficacy. Haemophilia. 2013;19:691-697.

19. Higgins JPT, Green S (editors). Cochrane Handbook for Systematic Reviews of Interventions Version 5.1.0 [updated March 2011]. The Cochrane Collaboration, 2011. Available from http://handbook. cochrane.org/. Accessed on May 5, 2016.

20. Signorovitch J, Swallow E, Kantor E, et al. Everolimus and sunitinib for advanced pancreatic neuroendocrine tumors: a matching-adjusted indirect comparison. Exp Hematol Oncol. 2013;2:32.
Journal of Blood Medicine

\section{Publish your work in this journal}

The Journal of Blood Medicine is an international, peer-reviewed, open access, online journal publishing laboratory, experimental and clinical aspects of all aspect pertaining to blood based medicine including but not limited to: Transfusion Medicine; Blood collection, Donor issues, Transmittable diseases, and Blood banking logistics; Immunohematology; Artificial and alternative

\section{Dovepress}

blood based therapeutics; Hematology; Biotechnology/nanotechnology of blood related medicine; Legal aspects of blood medicine; Historical perspectives. The manuscript management system is completely online and includes a very quick and fair peer-review system. Visit http://www.dovepress.com/ testimonials.php to read real quotes from published authors. 